\title{
PERFORMANCE HIDRÁULICA E PERFIL DE DISTRIBUIÇÃO DE ÁGUA DO MICROASPERSOR NAAN 7110, SOB DIFERENTES CONDIÇÕES DE VENTO'
}

\author{
José Dantas Neto², Mônica Garcia Agra de Medeiros ${ }^{3}$, \\ Carlos Alberto Vicira de Azevedo² e Hamilton Medeiros de Azevedo
}

\begin{abstract}
RESUMO
A principal finalidade de ensaios de equipamentos utilizados em sistemas de irrigação localizada consiste na determinação das características hidráulicas e nas análises de seu desempenho. Com o objetivo de fornecer subsídios ao correto dimensionamento de sistemas de irrigação por microaspersão, desenvolveu-se uma pesquisa sob condições de laboratório e de campo, em área pertencente ao Laboratório de Engenharia de Irrigação e Drenagem da Universidade Federal da Paraíba, com o fim de se avaliar a performance do microaspersor NAAN 7110. Em campo, trabalhou-se com velocidade do vento nas faixas de 0-2, 2-4, 4-6 e 6-8 km/h. Os testes visaram à determinação dos seguintes parâmetros: coeficiente de variação de fabricação; equação característica da relação entre pressão e vazão e diâmetro efetivo molhado médio em laboratório e campo. As análises mostraram que o microaspersor NAAN 7110 apresenta excelente uniformidade de fabricação, com Coeficiente de Variação de Fabricação (CVF) igual a 0,049. O melhor ajuste para a relação entre pressão e vazão foi dado pelo modelo do tipo potencial, com coeficiente de determinação 0,99 . Nas condições em que foram realizados os testes, não houve diferenciação entre os valores do diâmetro molhado médio obtidos em laboratório $(5,7 \mathrm{~m})$ e em campo $(5,7)$; entretanto, o diâmetro efetivo foi maior em campo $(5,7 \mathrm{~m})$ que em laboratório $(4,5 \mathrm{~m})$.
\end{abstract}

Palavras-chave: irrigação localizada, características hidráulica, diâmetro efetivo

\author{
HYDRAULIC PERFORMANCE AND WATER DISTRIBUTION \\ PROFILE OF 7110 NAAN MICROSPRINKLER \\ UNDER DIFFERENT WIND CONDITIONS
}

\begin{abstract}
The main purpose of testing equipments used in trickle irrigation systems consists of determining its hydraulic characteristics and analyzing its performance. Aiming to supply subsidies for an adequate design of trickle irrigation systems, a research was developed under laboratory and field conditions, in area belonging to the Irrigation and Drainage Engineering Laboratory at the of Paraíba, Federal University, in order to evaluate the performance of the microsprinkler NAAN 7110. In field, the emitter was tested for the wind speeds in the strips of $0-2,2-4,4-6$ and $6-8 \mathrm{~km} / \mathrm{h}$. The tests sought the determination of the following parameters: manufacturing variation coeflicient; characteristic equation of the relationship between pressure and discharge; and the average effective wetted diameter in laboratory and field. The analysis showed that the microsprinkler NAAN 7110 presents an excellent
\end{abstract}

1Parte da dissertação de Mestrado da segunda autora (DEAg/ (JFPb).

${ }^{2}$ Professor, PhD, Departamento de Engenharia Agrícola, Universidade Federal da Paraíba, Av. Aprigio Veloso, 882, Bodocongó, C.EP 58109-970, Campina Grande, PB, Brasil, E-mail: cazevedo@ deag.ufpb.br, Fone: (083)310.1318, Fax: (083)310.1184

${ }^{3}$ Eng Agronomo, mestranda em Eng Agrícola (DEAg/(JFPB). 
manufacturing uniformity, with a manufacturing variation coefficient (CVF) equal to 0,049. The best fitness for the pressure and discharge relationship was performed by the potential model, which presented a determination coefficient equal to 0,99 . Under the conditions in which the tests were performed, there was not differentiation between the values of the average wetted diameter obtained in laboratory $(5.7 \mathrm{~m})$ and in field $(5,7 \mathrm{~m})$. However, the eflective wetted diameter was larger in field $(5.7 \mathrm{~m})$ then in laboratory $(4.5 \mathrm{~m})$.

Key words: trickle irrigation, hydraulic characteristics, effective diameter

\section{INTRODUÇÃO}

A prática da irrigação, quando adequadamente utilizada, torna-se um eficiente instrumento na elevação da renda do produtor, economia de recursos, além da geração de empregos e, consequientemente, do aumento da oferta de produtos agrícolas. Um bom sistema de irrigação deve aplicar água no solo uniformemente, até determinada profundidade, proporcionando umidade necessária ao desenvolvimento normal das espécies vegetais.

Dentre os métodos de irrigação, a localizada vem sendo a mais utilizada nas regiões de maior escassez de água. O grande interesse despertado pelo sistema deve-se ao fato da sua aplicação, molhando apenas uma fração do sistema radicular das planlas favorecendo, assim, a economia de água e o aumento na produção. Azevedo (1986) afirma que a irrigação localizada consiste na aplicação da água molhando apenas uma fração do sistema radicular das plantas. A área máxima molhada não deve ser superior a $55 \%$ da área sombreada pela planta, enquanto a área mínima molhada é de $20 \%$ nas regiōes úmidas e de $30 \%$ nas regiões de clima semi-árido.

Os dois principais sistemas em que se emprega o método de irrigação localizada são a microaspersão e o gotejamento. Na microaspersão o ar é responsável pela difusão da água e, como esta aplicação é sob a forma de pequenas gotículas, sua distribuição é sensivelmente afetada pela velocidade do vento, resultando na desuniformidade do perfil de distribuição. A distribuição da água caracteriza todo o sistema de irrigação e esta tem efeitos diretos tanto no projeto como no seu funcionamento.

Para avaliação das diferenças individuais entre os emissores o melhor parâmetro indicado é o coeficiente de variação de fabricação (CVF). Por mais precisos que sejam os processos de fabricação dos emissores, é impossível se fabricar duas peças exatamente iguais. As pequenas diferenças entre dois emissores, os quais aparentemente são idênticos, podem causar variações significativas na vazão do sistema. Segundo Von Bewrmuth \& Solomom (1986) essas variações são decorrentes dos vários processos envolvidos na fase de fabricação. Dentre as causas mais comuns estão: dificuldades devidos à pressão e temperatura de moldagem que garantam confecção de dimensões críticas da passagem do lluxo; fabricação do molde, con liguração e variação do material usado na fabricação do emissor.

Os critérios de classilicação dos emissores, em função do coeficiente de variação de fabricação (CVF), são os apresentados na Tabela 1.

Keller \& Karmeli (1975), Von Betmuth \& Solomom (1986) e Abreu et al. (1987) sugerem a função potencial como a equação que descreve as características de vazão versus pressão do emissor, cuja a vazão se relaciona diretamente com a carga hidráulica atuante na entrada do emissor.

Para Keller \& Karmelli (1975) o expoente "x" caracteriza o regime de fluxo e a relação vazão versus pressão do emissor, de modo que:

$0<\mathrm{x}<0,5 \Rightarrow \mathrm{O}$ regime de escoamento varia de turbulento a totalmente turbulento, e a vazão sofre menos influência da variação da pressão.

$0,5<\mathrm{x}<1,0 \Rightarrow$ O regime de escoamento varia de instável a laminar, verificando-se maior influência das variações de pressão sobre a vazão.

De acordo com Azevedo (1986) e Abreu el al. (1987) para $x$ igual a zero a vazão é conslante, ou seja, independente da variação de pressão, tornando o emissor auto-compensante, que é uma condição ideal por permilir grandes variações de carga nas laterais, provocadas pela fricção e/ou pelo aclive do terreno.

O desempenho hidráulico do emissor é determinado pelo expoente $\mathrm{x}$, que é uma medida da variação do fluxo em resposta às variações de pressão; pelo coeficiente de variação de fabricação, que é uma medida da variação do lluxo causada pela variação no processo de fabricação e pela sensibilidade à temperatura, que é dependente do tipo de fluxo do emissor (Lima, 1991).

O que caracteriza e determina o funcionamento do microaspersor sobre uma superfície irrigada é o ensaio de distribuição pluviométrica. As curvas pluviométricas nos indicam a precipitação horária que recebe o terreno, enquanto as isoietas delimitam a área molhada, por diferentes precipitações.

Sadan e Shani (1983) citados por Armoni (1986) levantam a questão de que até que ponto pode uma área ser considerada irrigada ou simplesmente úmida e propõem um índice de precipitação inferior a 1,0 mm/h, como área úmida (irrigada ineficientemente); no entanto, concordam com o fato de que, para emissores com precipilação maior que $7 \mathrm{~mm} / \mathrm{h}$, um limite concreto seja inadequado, recomendando a definição de raio efetivo como sendo a distância entre o emissor e o pluviômetro que contenha $10 \%$ da pluviometria média com o emissor operando a $200 \mathrm{~K} \mathrm{~Pa}$, cuja definição lambém é dada por Abreu et al. (1987).

Este estudo teve a finalidade de avaliar a performance do microaspersor NAAN 7110, através de suas características hidráulica, e o perfil de distribuição em condições de laboratório e campo.

\section{MATERIAL E MÉTODOS}

Os ensaios foram realizados a nível de laboratório e campo na área experimental do Laboratório de Engenharia e 
Drenagem do Departamento de Engenharia Agrícola do Centro de Ciências e Tecnologia da Universidade Federal da Paraíba (LEID/DEAg/CCT/UFPB, em Campina Grande, PB, cujas coordenadas geográlicas são: latitude $7^{\circ} 13^{\prime} \mathrm{S}$, longitude $35^{\circ}$ $53^{\prime}$ W e altitude $547,56 \mathrm{~m}$. Na região a temperatura média normal do ar é de $22,5^{\circ} \mathrm{C}$, com umidade relativa do ar de $83 \%$ e velocidade do vento de $3,5 \mathrm{~m} / \mathrm{s}$.

O modelo de emissor estudado foi o microaspersor NAAN 7110, para se observar suas características hidráulicas e o funcionamento, no sentido de se solucionar possíveis problemas no dimensionamento de projetos. As vazões nos testes foram medidas através de leitura direta, sendo colocada, sobre o microaspersor, uma cuba que interceptava o jato d'água, o qual era coletado por um recipiente com capacidade de $580 \mathrm{ml}$, para posterior pesagem, a lim de se encontrar o volume a intervalo de tempo de 1 minuto. A medida do tempo era feito por meio de um cronômetro com precisão de 0,1 segundo e a pesagem através de uma balança eletrônica, com precisão de 0,1 grama.

Todas as medidas de pressão foram realizadas através de um manômetro de mercúrio com escala de $0,66 \mathrm{kPa}$ (inferior aos 2 Kpa exigidos pela ABNT, 1986) conectando-se a linha de lestes distante $30 \mathrm{~cm}$ da conexão do emissor; este manômetro é um tubo de plástico transparente, com diâmetro de $5 \mathrm{~mm}$.

Para se avaliar as variações que ocorrem na vazão entre os microaspersores, devido à variação durante o processo de fabricação, foram selecionados, ao acaso, 20 microaspersores novos, de acordo com a ABNT (1986).

Para determinação da vazão, cada microaspersor foi submetido a uma pressão de serviço de $200 \mathrm{kPa}$, sendo colocada, sobre o emissor, uma cuba capaz de interceptar o jato d'água, que era coletado através de um recipiente, em um espaço de tempo de $1 \mathrm{~min}$; os valores coletados foram posteriormente convertidos em $(1 / \mathrm{h})$, resultado da média de três repetições. A partir desses dados calcularam-se o desvio padrão e a vazão média; em seguida, determinou-se o coeficiente de variação de fabricação, dividindo-se o desvio-padrão pela média da vazão.

Após determinação do CVF, selecionaram-se seis microaspersores, que obtiveram vazões mais próximas da média para, em seguida, determinar as demais características do microaspersor.

Para a determinação da vazão, os microaspersores selecionados foram submetidos a diferentes pressões, medindose as respectivas vazões com três repetições, pelo método direto, tal como descritos anteriormente para determinação do CVF. As pressões de serviços utilizadas para determinação da relação vazão - pressão, foram: 50, 100, 150, 200, 250 e $300 \mathrm{kPa}$.

Foi determinada a curva característica vazão - pressão a partir dos resultados obtidos nos testes e, com esses mesmos dados, foi determinada a equação que relaciona vazão e pressão, de acordo com ABREU el al. (1987) segundo a expressão:

$$
\mathrm{q}=\mathrm{kh}^{\mathrm{x}}
$$

onde: $\mathrm{q}=$ vazão do emissor, $1 / \mathrm{h} ; \mathrm{k}=$ constante de proporcionalidade que caracteriza cada emissor; $h=$ pressão hidráulica determinada na entrada do emissor, $\mathrm{kPa} ; \mathrm{x}=$ expoente de emissor, parâmetro que caracteriza o fluxo de um emissor como função da pressão de operação.

Com ausência e presença de vento, este ensaio foi realizado com a finalidade de se determinar o raio efelivo e a distribuição pluviométrica. A velocidade do vento foi determinada através de um anemômetro instalado a uma altura de $50 \mathrm{~cm}$ e distante $10 \mathrm{~m}$ do experimento. A predominância do vento durante o experimento foi na direção Sudoeste.

Para avaliação desses parâmetros, foram instaladas duas linhas de pluviômetros dispostos orlogonalmente, com o emissor instalado na sua interseção (Figura 1). Os pluviômetros utilizados foram recipientes cilíndricos metálicos, medindo $8,4 \mathrm{~cm}$ de diâmetro e $10,5 \mathrm{~cm}$ de altura. Seguindo-se as normas da ABNT, os coletores foram espaçados equidistantes a cada $30 \mathrm{~cm}$.

Após 1 hora de funcionamento, com o emissor submetido a uma pressão de $200 \mathrm{kPa}$, foram feitas as leituras dos volumes de água contida em cada coletor, através da pesagem e, em

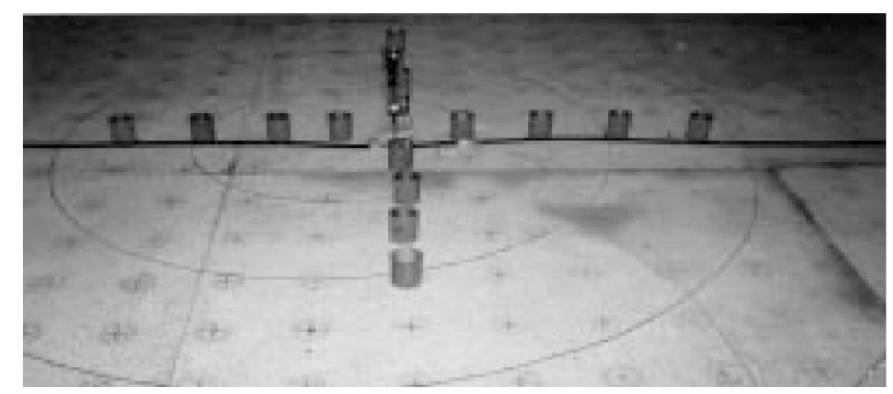

Figura 1. distribuição dos pluviômetros.

seguida, os volumes foram convertidos em altura de precipilação, e obtida a média dos 6 microaspersores ensaiados.

No início e no final de cada teste foi realizada a medição de vazão do emissor, com a finalidade de se observar a variação de vazão ao longo do tempo de funcionamento. Após determinação das precipitações médias obtidas nos testes, foi determinado o perfil pluviométrico do microaspersor estudado, com seu respectivo raio efetivo.

\section{RESULTADOS E DISCUSSÃO}

A temperatura da água nos testes ficou em torno de $25^{\circ} \mathrm{C}$, cuja massa específica é de $0,997 \mathrm{~g} / \mathrm{cm}^{3}$; no entanto, para transformação dos valores coletados em peso, para volume, considerou-se a massa específica igual a $1,0 \mathrm{~g} / \mathrm{cm}^{3}$. Dividindose o volume pela área do coletor $\left(0.0554 \mathrm{~m}^{2}\right)$ obliveram-se os valores médios de precipilação $(\mathrm{mm} / \mathrm{h})$ de cada microaspersor.

Com uma pressão de ensaio de $200 \mathrm{kPa}$, a média geral dos microaspersores foi de $39,8 \mathrm{l} / \mathrm{h}$, com um desvio-padrão de 0,195 l/h, e coeficiente de variação de fabricação (CVF) de 0.0049. De acordo com a ASAE, citado por KELLER \& BLIESNER (1990) o microaspersor estudado é considerado excelente do ponto de vista do processo de fabricação. Os valores típicos dos emissores encontrados no mercado apresentam, geralmente, coeficiente de fabricação variando entre 0,02 e 1,0 (Tabela 1).

A ABNT (1986) considera um emissor como bom aquele que apresenta coeficiente igual a 0,10 , o que equivale dizer que o CVF encontrado $(0,0049)$ tem precisão de 20 vezes maior que a recomendada por essa norma.

Na Tabela 2 encontram-se os valores de vazão média e 
os coeficientes de variação de fabricação nas diferentes pressões testadas. Observa-se, na citada Tabela que todos os valores dos coeficientes de variação de fabricação foram inferiores a $5 \%$ sendo, deste modo, classificados como excelentes, de acordo com a ABNT (1986). A vazão média encontrada para as três pressões estudadas corresponde a $97 \%$ da vazão fornecida pelo fabricante do microaspersor NAAN 7110.

Tabela 1. Critérios de classilicação dos emissores, em lunção do coeficiente de variação de fabricação (Segundo a ASAE cilado por Keller \& Bliesnce 1990)

\begin{tabular}{cc}
\hline Coeficiente de variação fabricação de $(\mathrm{CV})$ & Classificação \\
\hline$\leq 0,05$ & Excelente \\
$0,05-0,07$ & Médio \\
$0,07-0,11$ & Marginal \\
$0,11-0,15$ & Deficiente \\
$>0,15$ & Intaceilável
\end{tabular}

Tabela 2. Coelicientes de variação de labricação (CVF) em lunção das pressões testadas

\begin{tabular}{|c|c|c|c|c|}
\hline P'ressäo (kla & 150 & 200 & 250 & Média geral \\
\hline Vazảo delernuinada (l/h) & 35,29 & 39.80 & 44,62 & 39,90 \\
\hline $\mathrm{CVF}$ & 0,0046 & 0,0049 & 0,0044 & 0,0046 \\
\hline
\end{tabular}

Na Tabela 3 comparou-se os valores médios de vazão obtidos para as pressões estudadas e as vazões fornecidas pelo fabricante.

De modo geral, conforme se observa na coluna do erro sistemático, as vazões experimentais determinadas em laboratório estão próximas das fornecidas pelo catálogo do microaspersor NAAN 7110, sendo que, na pressão de serviço (200 kPa) a qual deve trabalhar o emissor, apresenta uma vazão apenas $2,7 \%$ menor que a vazão teórica. O catálogo do fabricante fornece pressões a partir de $100 \mathrm{kPa}$.

Tabela 3. Valores médios de vazão $(1 / h)$ dos microaspersores oblidos a partir de pressões crescentes.

\begin{tabular}{cccc}
$\begin{array}{c}\text { Pressĩo } \\
(\mathrm{kPa})\end{array}$ & $\begin{array}{c}\text { Vacão lionecida } \\
(1 / \mathrm{h})\end{array}$ & $\begin{array}{c}\text { Vazáo Lxperimental } \\
(1 / h)\end{array}$ & $\begin{array}{c}\text { Lrro Sistemático } \\
(\%)\end{array}$ \\
\hline 50 & - & 19,20 & - \\
100 & 29,00 & 27,80 & 4,1 \\
150 & 36,00 & 34,37 & 4,5 \\
200 & 41,00 & 39,90 & 2,7 \\
250 & 46,00 & 44,67 & 2,9 \\
300 & 50,00 & 49,08 & 1,8 \\
\hline
\end{tabular}

A partir dos dados de pressão e vazão determinados em laboratórios, apresentados na Tabela 3 , confeccionou-se a curva característica do microaspersor NAAN 7110 (Figura 2) com a respectiva equação característica; a equação é do tipo potencial $\left(\mathrm{Q}=2,4854 * \mathrm{~h}^{0,5235}\right)$. O mesmo modelo de equação foi encontrado por outros autores como: Botrel et al. (1985), Paes et al. (1986) e Alves \& Lima (1994)

O modelo potencial correlacionado apresentou alto coeficiente de determinação 0,99 , ou seja, $99 \%$ da variação da vazão são devidos à relação que existe entre a vazão e a pressão e a diferença se deve ao erro experimental.

$O$ expoente " $\mathrm{x}$ " da equação característica igual a 0,5235 caracteriza, segundo Keller \& Karmelli (1975), o emissor como

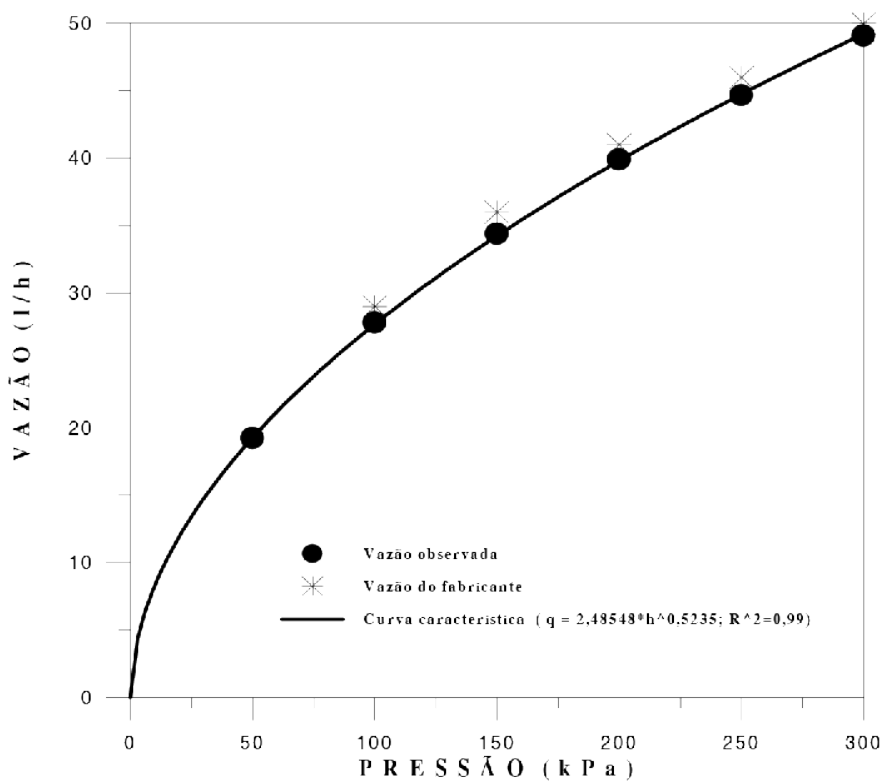

Figura 2. Relação entre pressão de serviço e vazão do microaspersor NAAN 7110 .

sendo de fluxo turbulento, significando que as mudanças na vazão, se relacionam com a raiz quadrada da pressão e, consequentemente, uma variação na energia de pressão da ordem de $20 \%$ permite que a vazão dos emissores varie em torno de \pm $10 \%$ em relação ao valor desejado. Botrel et al. (1985) encontrou " $\mathrm{x}$ " igual a 0,54 e Paz (1986) " $\mathrm{x}$ " igual a 0,55, respectivamente, para o microaspersor Dantas MA-120.

Para as diversas faixas de velocidade de vento estudadas, os valores médios de precipitação, para a pressão de $200 \mathrm{kPa}$ estão apresentados na Tabela 4. Observa-se que, à medida que aumenta a velocidade do vento, diminui a precipitação média do microaspersor.

A precipilação média encontrada em condições de campo $(1,85 \mathrm{~mm} / \mathrm{h})$ foi menor que a encontrada em condições de laboratório $(2,0 \mathrm{~mm} / \mathrm{h})$; em laboratório, até a distância de $135 \mathrm{~cm}$ do eixo do emissor, as precipilações coleladas foram superiores ao valor médio em todos os semi-eixos e, apesar

Tabela 4. Precipitações médias coletadas em condições de laboratório e em campo, para a pressão de $200 \mathrm{kPa}$

\begin{tabular}{cc}
\hline Condições da realização do teste & Médias de Precipitação $(\mathrm{mm} / \mathrm{h})$ \\
\hline laboratório & 2,00 \\
Velocidade de vento: $0-2 \mathrm{~km} / \mathrm{h}$ & 2,45 \\
Velocidade de vento: $2-4 \mathrm{~km} / \mathrm{h}$ & 1,83 \\
Velocidade de vento: $4-6 \mathrm{~km} / \mathrm{h}$ & 1,61 \\
Velocidade de vento: $6-8 \mathrm{~km} / \mathrm{h}$ & 1,53 \\
\hline
\end{tabular}

dos testes terem sido realizados na ausência de vento, houve maior precipitação no quadrante sudoeste, o que pode ser atribuído à configuração do microaspersor.

Na Figura 3 observa-se que, em condições de campo ocorreram, até a distância de $135 \mathrm{~cm}$, precipitações superiores à média para velocidades de vento de até $4 \mathrm{~km} / \mathrm{h}$ e, para velocidade de vento de 4 a $8 \mathrm{~km} / \mathrm{h}$, precipitações superiores a média, a uma distância de $105 \mathrm{~cm}$. Nas diferentes 
condições de vento a forma triangular do perfil de distribuição do microaspersor não foi alterada. O diâmetro médio molhado encontrado foi de $5,7 \mathrm{~m}$, tanto para os ensaios de laboratório como para os de campo. Em condições de laboratório Paes (1986) trabalhando com o microaspersor Dantas MA-120, encontrou diâmetro médio de 5,0m e Matos (1996) testando o microaspersor DAN SPRINKLER 2001, encontrou diâmetro de 4,5m. Sousa et. al. (1993) testando microaspersores em condições de campo, encontraram diâmetros médios de 5,7;6,0;6,8 e 6,0m, para velocidades de vento de 11,$52 ; 13,72 ; 13,90$ e $14,22 \mathrm{~km} / \mathrm{h}$, respectivamente. Afirmam os autores que a pouca diferença encontrada entre os valores dos diâmetros, é devida à pequena variação da velocidade do vento. Durante os ensaios em laboratório os recipientes colocados a uma distância de $225 \mathrm{~cm}$, totalizando um diâmetro efetivo de $450 \mathrm{~cm}$, estão incluídos na definição de diâmetro efetivo, ou seja, tiveram precipitação superior a $10 \%$ da precipitação média, o que corresponde a $75 \%$ do diâmetro teórico fornecido pelo fabricante. Para testes realizados em campo, todos os coletores colocados a uma distância de $285 \mathrm{~cm}$, obtiveram precipitação superior a $10 \%$ da precipitação média, correspondente a um diâmetro de $570 \mathrm{~cm}$.
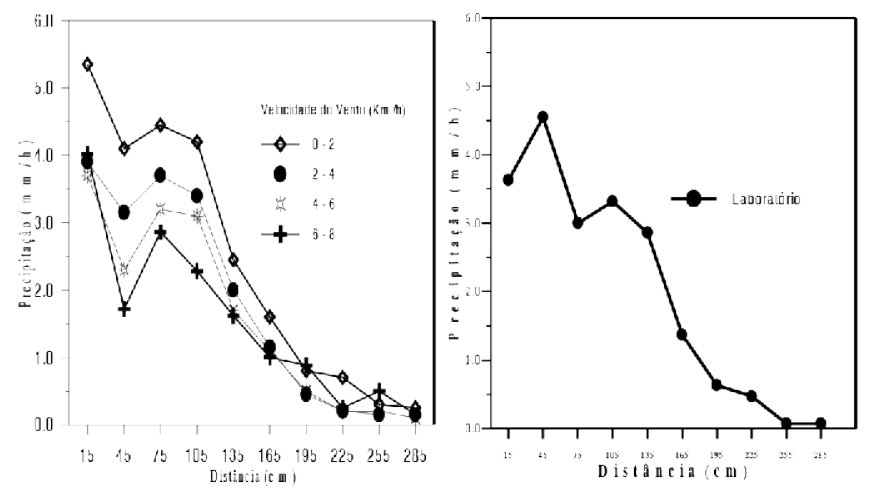

Figura 3. Semi-perfil médio do microaspersor NAAN 7110, em condições de campo e de laboratório.

\section{CONCLUSÕES}

Tendo-se como base os resultados obtidos através dos métodos usados na avaliação da performance do microaspersor NAAN 7110, e as condições em que os ensaios foram analisados, chegou-se às seguintes conclusões:

1. O microaspersor avaliado classifica-se como excelente segundo a $\mathrm{ABNT}$.

2. A intensidade de precipitação média encontrada em condições de campo $(1,85 \mathrm{~mm} / \mathrm{h})$ foi menor que a encontrada em condições de laboratório $(2,00 \mathrm{~mm} / \mathrm{h})$.

3. Nos testes de campo a velocidade do vento não influenciou no diâmetro molhado nem houve diferenciação entre o diâmetro médio molhado, em laboratório e em campo; entretanto, o diâmetro efetivo molhado foi maior em campo que em laboratório.

\section{REFERÊNCIAS BIBLIOGRÁFICAS}

ABREU, J.M.H.; LOPEZ, J.R.; REGALADO, A.P.; HERNANDEZ, J.F.G. El riego localizado. Madrid: Instituto Nacional de Investigaciones Agrárias, 1987, $317 \mathrm{p}$.

ALVES, D.R.D.; LIMA, L. A. Avaliação da uniformidade de distribuição de água de microaspersores e microdifusores. IN: CONGRESSO BRASILEIRO DE ENGENHARIA AGRÍCOLA, 23, 1994, Campinas. Resumo... Campinas: Associação Brasileira de Engenharia Agrícola, 1994, n. 94 - 3 - 316. 17p.

ARMONI, S. Micro - sprinkler irrigation. Kibuts Dan:Dan sprinklers, Israel, 1986, $91 \mathrm{p}$.

ASSOCIAÇÃO BRASILEIRA DE NORMAS TÉCNICAS - ABNT. São Paulo, Emissores para Sistema de Irrigação Iocalizada: Avaliação de Características Operacionais: Projeto 12: 02. 08. 21, São Paulo, 1986. $6 p$.

AZEVEDO, H.M. Irrigação localizada. Informe Agropecuário, Belo Horizonte, n.139,p. 40 - 53, 1986.

BOTREL, T.A.; OLITTA, A.F.L. ; OLIVEIRA, A. S. Hidráulica de microaspersores. O Solo. Piracicaba, n.77, p. 01-10, 1985.

KELLER, J. BLIESNER, R.D. Sprinkle and trickle irrigation. New York: Van Nostrand Reinhold, 1990, $651 \mathrm{p}$.

KELLER, J.; KARMELI, D. Trickle irrigation design. Glendora: Rain Bird splinkles manu-faturing corp. $1^{\text {a }}$ ed. $1975,133 p$.

LIMA, V. L.A. Caracterização hidráulica de tubulações laterais em microaspersão utilizando microtubos como dissipadores de energia. Campina Grande - UFPB, 1991. 11p. Dissertação (Mestrado em Engenharia Agrícola) - Universidade Federal da Paraíba, 1991.

MATOS, J.A. Características hidráulicas e da Distribuição de água do microaspersor DAN SPRINKIER 2001. Campina Grande - UFPB, 1996. 81p. Dissertação (Mestrado em Engenharia Agrícola). Universidade Federal da Paraíba, 1996.

PAES, L.AD.; LOREIRO, B.T.; BERNARDO, S.; FERREIRA, P.A Características hidráulicas do microaspersor "Dantas MA 120". Ceres,v.33, p309-319, 1986.

SOUSA, V. F.; BASTOS, E. S.; SILVA, A. A. G. S.; NETTO, A.O.A. Uniformidade de precipitação de microaspersores sob condição de campo. IN: CONGRESSO BRASILEIRO DE ENGENHARIA AGRÍCOLA, 22, 1993, Ilhéus. Resumo... Ilhéus: Associação Brasileira de Engenharia Agrícola, 1993, p.2493-2503.

VON BERNUTH, R; SOLOMON, K.H. Emilter constrution. IN: (NAKAYAMA, F.S.; BUCKS, D.A.). Trickle irrigation for crop producion. Phoenix, Chapler 2, p. $27-52.1986$. 